\title{
The association between serum uric acid and glaucoma severity in primary angle closure glaucoma: a retrospective case-control study
}

\author{
Shengjie Li ${ }^{1}$, Mingxi Shao ${ }^{1}$, Binghua Tang ${ }^{1}$, Aiping Zhang ${ }^{1}$, Wenjun Cao ${ }^{1,2}$, Xinghuai \\ Sun $2,3,4,5$ \\ ${ }^{1}$ Department of Clinical Laboratory, Eye and ENT Hospital, Shanghai Medical College, Fudan University, Shangai, China \\ ${ }^{2}$ Department of Ophthalmology and Visual Science, Eye and ENT Hospital, Shanghai Medical College, Fudan University, \\ Shangai, China \\ ${ }^{3}$ State Key Laboratory of Medical Neurobiology, Institutes of Brain Science and Collaborative Innovation Center for Brain \\ Science, Fudan University, Shanghai, China \\ ${ }^{4}$ Key Laboratory of Myopia, Ministry of Health (Fudan University), Shangai, China \\ ${ }^{5}$ Shanghai Key Laboratory of Visual Impairment and Restoration (Fudan University), Shangai, China \\ Correspondence to: Wenjun Cao, email: wgkjyk@aliyun.com \\ Xinghuai Sun, email: xhsun@shmu.edu.cn
}

Keywords: primary angle closure glaucoma, serum, uric acid, oxidative stress

Received: September 20, $2016 \quad$ Accepted: November 23, $2016 \quad$ Published: December 01,2016

\section{ABSTRACT}

Uric acid (UA) is a major antioxidant molecule and has been hypothesized to have a protective effect on the central nervous system against oxidative damage. We prospectively investigated the serum concentration of UA in primary angle closure glaucoma (PACG), and explored the association between serum concentration of UA and the severity of PACG. Using a retrospective case-control study design, 886 PACG subjects and 994 control subjects who attended the Eye \& ENT Hospital of Fudan University, were eligible for this study. Glaucoma severity was classified as mild $(M D \leq 6.00 \mathrm{~dB})$, moderate $(12 \mathrm{~dB} \geq M D>6 \mathrm{~dB})$ and severe ( $M D>12 \mathrm{~dB})$ based on the MD (mean deviation). The levels of UA were significantly lower $(p=0.025)$ in PACG $(0.286 \pm 0.082 \mathrm{mmol} / \mathrm{I})$ compared with control $(0.295 \pm 0.085 \mathrm{mmol} / \mathrm{I})$. The mean serum UA levels were lowest in the severe group $(0.281 \pm 0.074 \mathrm{mmol} / \mathrm{I})$ followed by moderate $(0.282 \pm 0.080 \mathrm{mmol} / \mathrm{I})$ and mild $(0.297 \pm 0.090 \mathrm{mmol} / \mathrm{I})$ with significant differences among the three groups $(p=0.032)$. In multivariate regression analysis, there was a significant negative correlation between UA level and vertical cup-disc ratio ( $B=-0.165, p=0.035)$. Significantly lower serum UA concentration in PACG and its negative association with disease severity presented it as an important candidate in reaction to oxidative stress in glaucoma pathogenesis.

\section{INTRODUCTION}

Primary glaucoma is a progressive optic neuropathy and one of the leading causes of global irreversible blindness, and the number of people (aged 40-80 years) with glaucoma worldwide is projected to increase from 76.0 million in 2020 and 111.8 million in 2040 [1-3]. Although chamber angle closure is known to be one of the key risk factors for PACG, oxidative stress and several other indispensible factors may also contribute [4-14].

As the final product of the common pathway of purine metabolism, UA is a major antioxidant with metalchelating properties [15] as well as the ability to scavenge nitrogen radicals and superoxide which help block the generation of strong oxidant peroxynitrite [16]. UA has been hypothesized to have a protective effect on the central nervous system against oxidative damage $[17,18]$. Lolekha P et al. [19] and Vieru E et al. [20] reported that patients with Parkinson's disease showed significantly lower serum UA and uric acid/creatinine (UA/Cr) ratio than control. Whether serum UA levels are changed and involved in the pathophysiological mechanisms of PACG remained unclear. Babizhayev MA et al. [21] reported that prevention of oxidative stress exposure to the trabecular meshwork with a $\mathrm{N}$-acetylcarnosine ophthalmic prodrug of carnosine and oral formulation of nonhydrolizedcarnosine may help to reduce the progression of glaucoma. Moreover, several studies suggested that 
markers of oxidative stress including chemia-modified albumin, protein nitrotyrosine, lipid oxidation products and 8-hydroxydeoxyguanosin, increased significantly in glaucoma [7, 22-25]. However, whether peripheral blood UA concentration and $\mathrm{UA} / \mathrm{Cr}$ ratio were changed in PACG and associated with the pathogenesis of glaucoma still remained to be studied.

The objective of this study was to measure serum UA and creatinine concentration and to further explore the relationships between $\mathrm{UA}$ and $\mathrm{UA} / \mathrm{Cr}$ ratio and glaucoma severity in PACG.

\section{RESULTS}

\section{Characteristics of the study patients}

A total of 886 PACG subjects and 994 control subjects from the Eye \& ENT Hospital of Fudan University were eligible for the study from January 2010 to December 2015. Only one eye was selected randomly if both eyes suffered from PACG. There was no statistical difference in the mean age and gender between the PACG and control group $(p>0.05)$. The mean serum levels of $\mathrm{UA}$ and $\mathrm{UA} / \mathrm{Cr}$ ratio were significantly lower in the PACG compared with the control group $(p<0.001)$. PACG group had a higher serum creatinine concentration compared with the control group $(p<0.001)$. The demographic, $\mathrm{Cr}$, $\mathrm{UA}, \mathrm{UA} / \mathrm{Cr}$ ratio of the PACG and control groups were summarized in Table 1.

\section{Comparison of $\mathrm{UA}$, creatinine, $\mathrm{UA} / \mathrm{Cr}$ ratio and ocular parameters in subjects with PACG, stratified according to severity}

Based on the MD, the PACG subjects were categorized into 3 subgroups of different severity level of which 286 were classified as mild, 198 as moderate and 402 as severe. There was no statistical difference in the mean age and gender ( $p=0.605, p=0.063$, respectively) among the three groups. The mean serum levels of UA was lowest in the severe PACG group, followed by moderate PACG and mild PACG, and the differences among groups were significant $(p=0.032)$. The mean serum level of creatinine was not significantly different among the three groups $(p>0.05)$. A similar trend was observed when UA were compared among the 3 groups with respect to gender. The ocular parameters were significantly different among the three groups $(p<0.05)$. The level of UA, creatinine, $\mathrm{UA} / \mathrm{Cr}$ ratio, and ocular characteristics of the three groups were shown in Table 2 and Figure 1.

\section{Spearman correlation between UA and ocular parameters}

Table 3 demonstrated the Spearman correlations of UA with the ocular parameters. In the overall PACG group, a statistically significant negative correlation between UA and VCDR (vertical cup-disc ratio) $(r=-0.111, p=0.001)$, and positive correlation between UA and AL (axial length) $(r=0.151, p<0.001)$ were observed. In the mild PACG group, a negative correlation between UA and VCDR $(r=-0.308, p<0.001)$, UA and MD $(r=-0.242, p=0.013)$, and positive correlation between UA and AL $(r=0.142, p=0.025)$, UA and MS (mean sensitivity) $(r=0.471, p<0.001)$ were observed. In the moderate PACG group, the correlations between UA and AL, UA and MD, UA and MS were also statistically significant. A similar trend was observed when correlation among UA and ocular parameters were performed with respect to gender.

\section{Multiple linear regressions for associations between $\mathrm{UA}$ and ocular parameters}

Table 4 demonstrated the multiple linear regressions of UA with the ocular parameters. In multivariate regression analysis after adjusting for age, gender, blood pressure, and BMI, there was a statistically significant negative correlation between UA and VCDR $(B=-0.165$, $p=0.035)$ in the overall PACG patients. In the mild PACG group, a negative correlation between UA and VCDR $(r=-0.156, p=0.026)$, and positive correlation between UA and MS ( $r=0.341, p=0.046)$ were observed. In the severe PACG group, a positive correlation between UA and VCDR $(r=-0.150, p=0.044)$ was observed. A similar trend was observed when association among UA and ocular parameters were performed by multiple linear regressions with respect to gender.

\section{DISCUSSION}

To the best of our knowledge, this study has been the first to focus on the investigation of serum UA and $\mathrm{UA} / \mathrm{Cr}$ ratios in PACG and further evaluate their relationship with disease severity. The principal finding was that serum UA levels and $\mathrm{UA} / \mathrm{Cr}$ ratios in PACG patients were lower compared with sex and age-matched control group subjects, in addition, UA levels were found to be associated with the severity of PACG. The serum $\mathrm{UA} / \mathrm{Cr}$ ratio was used to reduce the possible interference caused by sex and differences in renal function [20]. These data suggested a possible role of UA in the pathogenesis of PACG.

The levels of peripheral blood UA have been reported to be associated with hypertension [26] and Alzheimer's disease [27]. Oxidative stress played a role in neuron cell death and contributes to the pathogenesis of these diseases [17]. Oxidative stress was also considered to contribute to the physiologic alterations in aqueous humor outflow and subsequently to IOP elevation and retinal ganglion cell (RGC) damage in glaucoma $[13,28]$. 
Table 1: Demographics, creatinine, UA, and UA/Cr ratio of subjects with PACG

\begin{tabular}{lcccc}
\hline \multicolumn{1}{c}{ Factors } & PACG group & Control group & $\boldsymbol{t}$ value & $\boldsymbol{P}$ value \\
\hline Age (year) & $63.17 \pm 10.65$ & $63.26 \pm 10.12$ & 0.199 & 0.842 \\
Gender (male/female) & $302 / 584$ & $370 / 624$ & 2.008 & 0.156 \\
Creatinine (umol/1) & & & 2.478 & 0.013 \\
Total & $69.54 \pm 17.61$ & $67.34 \pm 20.45$ & 3.009 & 0.003 \\
Male & $80.41 \pm 16.51$ & $77.84 \pm 19.85$ & 2.879 & 0.004 \\
Female & $63.92 \pm 15.39$ & $61.12 \pm 18.14$ & & 0.025 \\
UA (mmol/l) & & & 2.236 & 0.023 \\
Total & $0.286 \pm 0.082$ & $0.295 \pm 0.085$ & 2.274 & 0.049 \\
Male & $0.327 \pm 0.078$ & $0.342 \pm 0.088$ & 1.972 & $<0.001$ \\
Female & $0.260 \pm 0.068$ & $0.268 \pm 0.069$ & & $<0.001$ \\
UA/Cr ratio & & & 3.709 & $<0.001$ \\
Total & $4.23 \pm 1.20$ & $4.51 \pm 1.24$ & 3.716 & 3.703 \\
Male & $4.17 \pm 1.15$ & $4.53 \pm 1.31$ & $4.51 \pm 1.19$ & \\
Female & $4.25 \pm 1.23$ & & & \\
\hline
\end{tabular}

Abbreviations: UA: uric acid, Data are expressed as mean \pm standard deviation (SD).

Independent-Samples $T$ Test and chi-square test were used.

Table 2: Comparison of demographics, creatinine, $\mathrm{UA}, \mathrm{UA} / \mathrm{Cr}$ ratio and ocular parameters in subjects with PACG, stratified according to severity

\begin{tabular}{|c|c|c|c|c|}
\hline & Mild PACG, $n=286$ & Moderate PACG, $n=198$ & Severe PACG, $n=402$ & $P$ value \\
\hline Age, years & $62.79 \pm 10.57$ & $63.77 \pm 10.87$ & $63.14 \pm 10.61$ & 0.605 \\
\hline Female, N (\%) & $186(65.03)$ & $144(73.73)$ & $254(63.18)$ & 0.063 \\
\hline $\mathrm{SBP}(\mathrm{mm} \mathrm{Hg})$ & $131.40 \pm 14.82$ & $130.20 \pm 13.37$ & $131.01 \pm 14.60$ & 0.671 \\
\hline DBP (mm Hg) & $76.39 \pm 8.39$ & $76.54 \pm 7.95$ & $76.18 \pm 9.62$ & 0.891 \\
\hline $\mathrm{BMI}, \mathrm{Kg} / \mathrm{m}^{2}$ & $23.19 \pm 3.30$ & $22.45 \pm 2.92$ & $22.79 \pm 4.08$ & 0.285 \\
\hline IOP (mm Hg) & $28.80 \pm 10.55$ & $30.49 \pm 11.46$ & $36.78 \pm 13.11$ & $<0.001^{\mathrm{a}, \mathrm{c}}$ \\
\hline VCDR & $0.46 \pm 0.17$ & $0.59 \pm 0.20$ & $0.81 \pm 0.20$ & $<0.001^{\mathrm{a}, \mathrm{b}, \mathrm{c}}$ \\
\hline $\mathrm{CCT}(\mathrm{mm})$ & $547.52 \pm 55.21$ & $537.66 \pm 43.11$ & $543.91 \pm 50.66$ & 0.201 \\
\hline $\mathrm{ACD}(\mathrm{mm})$ & $1.80 \pm 0.46$ & $1.85 \pm 0.44$ & $1.85 \pm 0.45$ & 0.341 \\
\hline $\mathrm{AL}(\mathrm{mm})$ & $22.16 \pm 1.17$ & $22.29 \pm 0.94$ & $22.55 \pm 1.43$ & $0.001^{\mathrm{a}, \mathrm{c}}$ \\
\hline $\mathrm{MD}(\mathrm{dB})$ & $3.85 \pm 1.83$ & $9.20 \pm 2.15$ & $21.65 \pm 4.71$ & $<0.001^{\mathrm{a}, \mathrm{b}, \mathrm{c}}$ \\
\hline $\mathrm{MS}(\mathrm{dB})$ & $20.52 \pm 7.77$ & $17.83 \pm 2.79$ & $5.56 \pm 4.77$ & $<0.001^{\mathrm{a}, \mathrm{b}, \mathrm{c}}$ \\
\hline \multicolumn{5}{|c|}{ Creatinine (umol/1) } \\
\hline Total & $71.53 \pm 20.13$ & $68.70 \pm 16.18$ & $68.53 \pm 16.22$ & $0.065^{\mathrm{a}}$ \\
\hline Male & $85.22 \pm 21.10$ & $82.92 \pm 13.64$ & $76.29 \pm 12.51$ & $<0.001^{\mathrm{a}, \mathrm{b}}$ \\
\hline Female & $64.18 \pm 15.18$ & $63.50 \pm 13.75$ & $63.96 \pm 16.44$ & 0.921 \\
\hline \multicolumn{5}{|l|}{$\mathrm{UA}(\mathrm{mmol} / \mathrm{l})$} \\
\hline Total & $0.297 \pm 0.090$ & $0.282 \pm 0.080$ & $0.281 \pm 0.074$ & $0.032^{\mathrm{a}, \mathrm{b}}$ \\
\hline Male & $0.342 \pm 0.085$ & $0.322 \pm 0.058$ & $0.317 \pm 0.079$ & $0.040^{\mathrm{a}}$ \\
\hline Female & $0.273 \pm 0.083$ & $0.267 \pm 0.083$ & $0.258 \pm 0.061$ & $0.049^{\mathrm{a}}$ \\
\hline \multicolumn{5}{|l|}{$\mathrm{UA} / \mathrm{Cr}$ ratio } \\
\hline Total & $4.25 \pm 1.21$ & $4.23 \pm 1.29$ & $4.22 \pm 1.14$ & 0.948 \\
\hline Male & $4.15 \pm 1.14$ & $3.97 \pm 0.89$ & $4.24 \pm 1.23$ & 0.332 \\
\hline Female & $4.31 \pm 1.25$ & $4.33 \pm 1.40$ & $4.18 \pm 1.09$ & 0.384 \\
\hline
\end{tabular}

Abbreviations: UA: uric acid, SBP: systolic blood pressure, DBP: diastolic blood pressure, BMI: body mass index, IOP: intraocular pressure, VCDR: vertical cup-disc ratio, CCT: central corneal thickness, AL: axial length, ACD: anterior chamber depth, MD: visual fields mean deviation, MS: visual fields mean sensitivity, PACG: primary angle closure glaucoma. Data are expressed as mean \pm standard deviation (SD). Chi-square test and One-way ANOVA was used.

${ }^{a} P<0.05$ for the difference between Mild PACG and Severe PACG (1-way ANOVA with the LSD post hoc test).

${ }^{\mathrm{b}} P<0.05$ for the difference between Mild PACG and Moderate PACG (1-way ANOVA with the LSD post hoc test).

${ }^{\mathrm{c}} P<0.05$ for the difference between Moderate PACG and Severe PACG (1-way ANOVA with the LSD post hoc test). 
In this study, we found significantly lower serum UA levels in PACG patients compared with control group in both male and female subjects. However, Yuki K et al. [29] reported that higher serum uric acid levels was found in their normal tension glaucoma subjects in comparison to their control subjects. We speculate that the difference in disease etiology may explain the different findings. Erdurmus $\mathrm{M}$ et al. [30] and Ferreira SM et al. [31] reported that serum level of total antioxidant capacity was decreased in patients with primary open-angle glaucoma and pseudoexfoliation glaucoma. In addition, aqueous humor UA levels were significantly lower in the congenital glaucoma rabbit model compared with the agematched controls [32]. Sorkhabi R et al. [22] reported a significant correlation between a higher aqueous humor 8-hydroxydeoxyguanosin level and lower antioxidant capacity levels in the serum of patients with glaucoma. Therefore, the serum antioxidant capacity could reflect the local ocular redox status [33]. We suggest that the decreased level of serum UA may play a part in the antioxidative insufficiency which could contribute to PACG development.

There have been reports suggesting that glaucomatous damage is associated with episodes of systemic inflammation which are likely to cause a systemic decrease in UA [34]. Oxidative stress, inflammatory response, infection and cell death appear to be correlated. Oxidative stress induced an inflammatory response that interfered with the homeostasis of the cell [35]. Several inflammatory molecules such as IL-6, IL-8, IL-1alpha, ELAM-1 were up-regulated which may also be induced by oxidative stress [35]. Astafurov K et al. [34] reported that patients with glaucoma had higher bacterial oral counts compared to control subjects and lipopolysaccharide administration in glaucoma animal models resulted in enhancement of axonal degeneration and neuronal loss. In addition, some studies reported that infection of $\mathrm{H}$. pylori had a statistically significant association with glaucoma $[36,37]$. Being one of the main anti-oxidants, UA might be consumed by oxidizing agents to prevent an inflammatory response, as mentioned by the above studies. Therefore, we suspect that systemic inflammation and oxidative stress were likely to cause an obvious consumption of UA and significant decrease of its serum level.

Moreover, the decreased serum levels of UA in PACG patients were an outcome of an oxidative stressrelated process in the optic nerve system, eye tissue and possibly in other tissues which meant that this decrease was likely to be a result of the direct reaction between UA and oxidizing agents $[27,38]$. Sacca SC et al. [10] reviewed that oxidative free radicals and reactive oxygen species were able to affect the cellularity of the human trabecular meshwork. The above studies indicated that glaucoma patients were under a high oxidation state both in the eye and body. Therefore, it is possible that UA would be consumed in the process of PACG by preferentially reacting with oxidizing agents both in the eye and peripheral blood.

In vitro studies showed that oxidative stress accelerated cell apoptosis and led to accumulation of extra cellular matrix in the trabecular meshwork, which could both increase the resistance of the aqueous humor outflow and result in IOP elevation and later visual field loss $[23,39,40]$. Our previous work showed that chronic exposure of angular aqueousplexus cells to oxidative stress decreased cell monolayer permeability and upregulated cytoskeletal and cell-cell adhesion protein expression [14]. Hooper DC et al. [41] reported that mouse exhibited diminished signs (piloerection, tail paralysis, hind limb paralysis, moribund) and better survival in a multiple sclerosis disease model which was treated with UA injection. Scott GS et al. [42] reported that UA played
Total

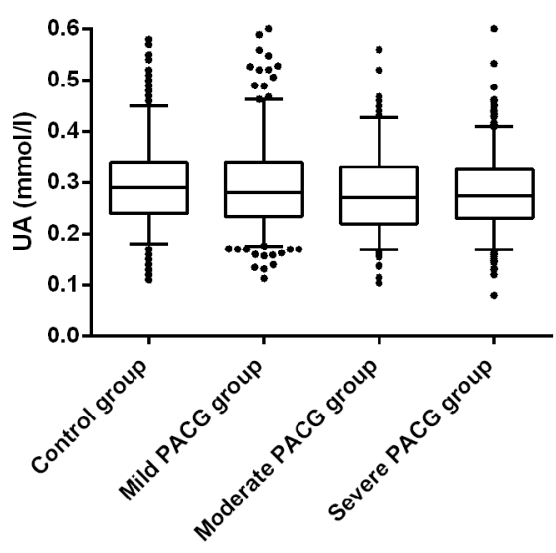

Male subgroup

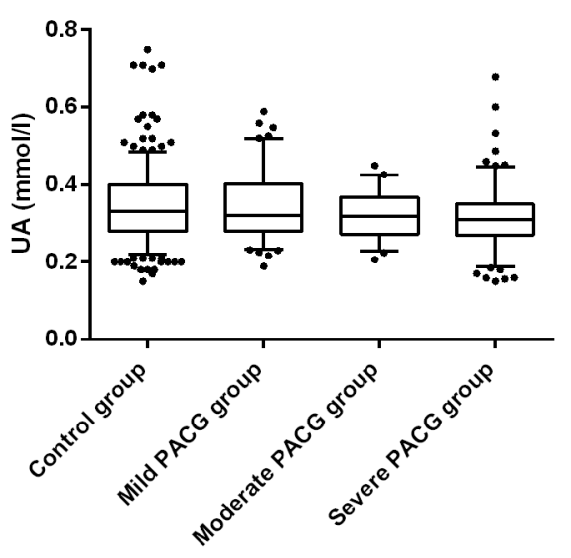

Female subgroup

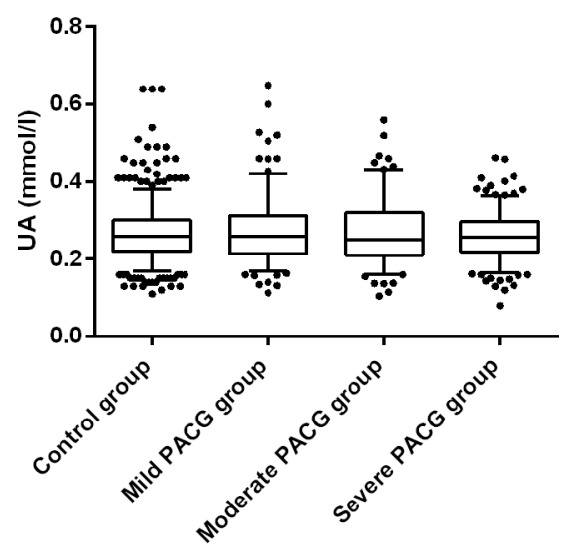

Figure 1: Comparison of serum levels of uric acid (UA) in patients with mild, moderate, severe PACG and control group: using box-and-whisker plot. The box contained $50 \%$ of all values (from 25 th to 75 th percentile) and was divided by the horizontal bar of the median value (50th percentile). The whiskers showed the remainder of the distribution (1.5 $\times$ Inter Quartile Range). Outliers were shown as dots. 
Table 3: Spearman correlation between UA and ocular parameters

\begin{tabular}{|c|c|c|c|c|c|c|c|c|c|}
\hline & \multicolumn{4}{|c|}{ Spearman Correlation ( $p$ value) } & \multicolumn{2}{|c|}{$\begin{array}{c}\text { Male PACG Spearman Correlation } \\
(p \text { value })\end{array}$} & \multicolumn{3}{|c|}{ Female PACG Spearman Correlation ( $p$ value) } \\
\hline & Overall & Mild & Moderate & Severe & Mild & Severe & Overall & Mild & Moderate \\
\hline VCDR & $-0.111(0.001)$ & $-0.308(<0.001)$ & NS & $-0.136(0.009)$ & $-0.371(<0.001)$ & $-0.214(0.011)$ & $-0.169(<0.001)$ & $-0.211(0.005)$ & NS \\
\hline $\mathrm{AL}$ & $0.151(<0.001)$ & $0.142(0.025)$ & $0.233(0.002)$ & NS & NS & NS & NS & NS & $0.182(0.039)$ \\
\hline MD & NS & $-0.242(0.013)$ & $-0.169(0.047)$ & NS & $-0.308(0.050)$ & NS & NS & NS & NS \\
\hline MS & NS & $0.471(<0.001)$ & $0.209(0.014)$ & NS & $-0.549(<0.001)$ & NS & NS & $0.305(0.016)$ & $0.226(0.020)$ \\
\hline
\end{tabular}

Abbreviations: UA: uric acid, VCDR: vertical cup-disc ratio, AL: axial length, MD: visual fields mean deviation, MS: visual fields mean sensitivity, NS: not significant. PACG: primary angle closure glaucoma. Male PACG Spearman correlation: no statistically significant correction between UA and ocular parameters in overall and moderate subgroup. Female PACG Spearman correlation: no statistically significant correction between UA and ocular parameters in severe subgroup.

Table 4: Multiple linear regressions for associations between UA and ocular parameters

\begin{tabular}{|c|c|c|c|c|c|c|c|c|c|}
\hline & \multicolumn{4}{|c|}{ PACG B ( $p$ value) } & \multicolumn{2}{|c|}{ Male PACG B ( $p$ value) } & \multicolumn{3}{|c|}{ Female PACG B ( $p$ value) } \\
\hline & Overall & Mild & Moderate & Severe & Mild & Severe & Overall & Mild & Moderate \\
\hline VCDR & $-0.165(0.035)$ & $-0.156(0.026)$ & NS & $-0.150(0.044)$ & NS & $-0.284(0.020)$ & $-0.209(0.040)$ & $-0.420(0.001)$ & NS \\
\hline $\mathrm{AL}$ & NS & NS & $0.259(0.025)$ & NS & NS & NS & NS & NS & NS \\
\hline MS & NS & $0.341(0.046)$ & NS & NS & $-0.502(0.020)$ & NS & NS & NS & $0.239(0.049)$ \\
\hline
\end{tabular}

Abbreviations: UA: uric acid, VCDR: vertical cup-disc ratio, AL: axial length, MS: visual fields mean sensitivity, NS: not significant. PACG: primary angle closure glaucoma. Analysis adjusted for age, gender, blood pressure, and BMI. Male PACG: no statistically significant correction between UA and ocular parameters in overall and moderate subgroup. Female PACG: no statistically significant correction between UA and ocular parameters in severe subgroup.

an important role in the protection against peroxynitritemediated pathogenesis of inflammatory CNS disorders. In cellular models of neuronal death induced by glutamate exposure or oxygen-glucose deprivation, the exogenous administration of UA reduced neuronal damage, suppressing the accumulation of ROS [43]. Therefore, UA has been hypothesized to have a protective effect on the central nervous system against oxidative damage [17, 44]. These results led us to speculate that lower levels of UA may be risk factors of glaucomatousoptic neuropathy.

Moreover, based on the MD, the PACG subjects were categorized into 3 subgroups of different severity level (mild, moderate, and severe). The mean serum levels of UA were lowest in the severe PACG group, followed by moderate PACG and mild PACG. However, the mean serum level of UA was not statistically different between moderate and severe PACG group (Figure 1 and Table 2). This suggests that UA might play an important role in the beginning of the disease process, and the role of the UA decreases in more advanced stages of the disease. There were also statistically significant negative correlations between UA and VCDR, UA and MD, and a positive correlation between UA and MS. Since the correlation may also be affected by other factors, multivariate analysis was conducted to further investigate the association between UA and glaucoma. Statistical data showed that there was also a significant correlation between UA and VCDR, and between UA and MS. We speculated that the oxidative stress system was increasingly activated along with the progression of PACG while the serum level of UA decreased accordingly. In conclusion, we hypothesize that the mean serum level of UA was associated with PACG severity. This predictive association between serum UA level and PACG severity further indicated the potential role of UA as an important anti-oxidative content in the disease pathogenesis. This finding was novel and potentially very important. The exploration for the role of UA and oxidative stress in PACG appeared to be quite difficult. The mechanism of UA in the pathogenesis of glaucomatous optic neuropathy was worth further exploration in our follow-up study.

Moreover, Table 1 highlighted significant difference in UA between PACG and control groups. We found that the difference between men in PACG and control groups in UA levels was only 0.015 . However, the difference between females and males was much higher: in the PACG group it was 0.067 (4-fold higher); in the control group it was 0.074 (5-fold higher). Recently, population-based surveys of glaucoma in Asians reported that the prevalence of PACG was higher in the female than male population $[45,46]$. Cheng JW et al. [46] found that PACG affects women 1.51 times more frequently than men. Therefore, our hypothesis was that the much lower UA level might be the cause for the female to have a much higher risk of PACG.

Although this is the first study which focused on the evaluation of serum level of UA, UA/Cr ratios and its relationship between PACG and disease severity, we acknowledged that our present study has some limitations. (1) We did not consider treatment with brinzolamide eye drops, carbonic anhydrase inhibitor, and mannitol and how it might affect renal function. In this study, there was no statistical difference of creatinine among the mild, moderate, and severe PACG subgroups. The serum UA/Cr ratio was analyzed considering the existence of possible interference such as medication and difference in renal function. $\mathrm{UA} / \mathrm{Cr}$ ratios in PACG patients were also lower 
compared with the control group. (2) Owing to this study was a retrospective case-control study, information on ocular parameters in controls subjects was lacking.

The present study demonstrated that the mean serum level of UA was decreased and negatively associated with PACG severity which suggested the possible association between UA with the development of glaucoma and the involvement of oxidative stress in the pathogenesis of PACG.

\section{MATERIALS AND METHODS}

\section{Patients}

This was a retrospective case-control study design. The study was approved by the Ethics Committee of the Eye-ENT Hospital of Fudan University, Shanghai, China and was conducted according to the Declaration of Helsinki. Written informed consent for the use of any clinical data in research was obtained for all patients at the time of admission to the Eye-ENT Hospital of Fudan University. PACG subjects and control subjects who attended the Eye-ENT Hospital of Fudan University from January 2010 to December 2015 were selected according to the inclusion criteria listed below.

\section{Recruitment procedures}

The data of all the PACG subjects $(n=1350)$ during January 2010 through December 2015 and control subjects $(n=1645)$ were collected from the inpatient electronic database. 446 PACG subjects and 595 control subjects were excluded according to the inclusion criteria. The exclusion rate of PACG and control group was $33.04 \%$ and $36.17 \%$ respectively. A further 56 control subjects were randomly excluded because they were not age and sex matched to the PACG group. At last, a total of 886 PACG subjects and 994 control subjects from the Eye \& ENT Hospital of Fudan University were eligible for the study.

\section{Inclusion criteria}

\section{PACG subjects}

(1) PACG subjects were selected from inpatients. Each subject underwent a standardized ophthalmic examination, which included refractive status, slit-lamp biomicroscopy, fundus examination, IOP, CCT (central corneal thickness), AL, ACD (anterior chamber depth), visual field examination, and gonioscopy, performed by glaucoma specialists. MD (visual field-derived mean deviation) and MS (mean sensitivity) were measured by Octopus automated perimetry (HAAG, STREIT, Switzerland). IOP was measured using Goldmanapplanation tonometry. Fundus photography was performed with a retinal camera (TRC-NW200, Topcon). A-scan ultrasound (A-Scan Pachymeter, Ultrasonic, Exton, PA, USA) was used to measure AL, ACD, and CCT.
(2) PACG was diagnosed on the basis of narrow angles with glaucomatous optic neuropathy with corresponding visual field loss. This was defined as glaucomahemifield test outside normal limits including a cluster of three or more, non-edge, contiguous points on the pattern deviation plot, not crossing the horizontal meridian with a probability of less than $5 \%$ of being present in the age-matched normal (one of which was less than $1 \%$ ), an abnormal pattern standard deviation with $\mathrm{P}$ less than $5 \%$ occurring in the normal population, and fulfilling the test reliability criteria (fixation losses less than $20 \%$, false positives less than $33 \%$ and/or false negatives less than 33\%). PACG was diagnosed in eyes with narrow angles, with elevated IOP (IOP $>21 \mathrm{~mm} \mathrm{Hg}$ ); at least 180 of angle-closure obliterating the pigmented part of the trabecular meshwork, whether synechial or appositional, segmented or continuous; and eyes in which the degree of peripheralanteriorsynechial is too extensive to be managed by laser peripheral iridotomy. [4, 47, 48] Newly diagnosed PACG patients and referral PACG patients were included. Subjects receiving glaucoma medications were also included. Patients who were taking medications which influence serum uric acid level were excluded. Patients were divided into three groups with different severity based on $\mathrm{MD}$, i.e. mild (MD $\leq 6.00 \mathrm{~dB})$, moderate $(12 \mathrm{~dB}$ $\geq \mathrm{MD}>6 \mathrm{~dB})$ and severe $(\mathrm{MD}>12 \mathrm{~dB})[49,50]$.

(3) PACG subjects had no other ocular diseases and systemic diseases such as hyperuricemia, diabetes, cardiac, autoimmune disease and cancer.

\section{Control subjects}

Control subjects were composed of patients with strabismus $(n=168)$, amblyopia $(n=146)$, nystagmus ( $n=111)$, epicophosis $(n=144)$, vocal cords leukoplakia $(n=163)$, lacrimal ducts obstruction $(n=155)$, and nasal septal deviation $(n=107)$. They were selected from the inpatients record at the Eye-ENT Hospital of Fudan University from January 2010 to December 2015. Each subject underwent a standardized physical examination and preliminary ophthalmic examinations, which included refractive status, slit-lamp, and IOP, performed by glaucoma specialists. IOP was measured using Goldmanapplanation tonometry. All the control subjects were excluded from any family history of glaucoma and systemic diseases which included but not restricted to hyperuricemia, diabetes, cardiac, autoimmune disease and cancer.

\section{UA and creatinine}

In this study, PACG and control subjects were selected from the hospital inpatients. As part of a standard care at the Fudan's University Eye \& ENT Hospital, peripheral blood samples were routinely collected from all the inpatients and tested for UA levels. Quantification of serum UA and creatinine was measured by enzymatic 
colorimetry using a commercially available kit (Roche Diagnostics GmbH, Mannheim, Germany). Internal controls were analyzed daily over the 6-year period, with typical monthly CVs of $2 \%-4 \%$ and no significant changes in the values. The reference ranges for UA between males and females were different $[51,52]$. The reference range of UA was $0.2023-0.4165 \mathrm{mmol} / 1$ for males and 0.1428 $0.3392 \mathrm{mmol} / 1$ for females. Therefore, the subjects were categorized into gender subgroups.

\section{Data analysis}

The data was analyzed by SPSS13.0 (SPSS Inc., Chicago, IL). UA and creatinine concentrations were presented as mean \pm standard deviation (SD). UA/Cr ratio $=\left(\mathrm{UA}^{*} 1000\right) / \mathrm{Cr}$. Normality was assessed with the Kolmogorov-Smirnoff test. The independent student's $t$-test and chi-square test were used for comparison of characteristics of patients between the PACG group and control group. The one-way ANOVA test was used to compare the levels of serum UA, serum creatinine, UA/ $\mathrm{Cr}$, and ocular parameters among the three groups with different severity. The associations between UA and ocular parameters in PACG were analyzed by Spearman correlation. After Spearman correlation, multivariate linear regression analysis (adjusting for age, gender, blood pressure, and BMI (body mass index) to prevent bias) was performed to evaluate the association between UA and disease severity, namely visual field indices (MD, MS), VCDR, and other ocular parameters. A $P$ value of less than 0.05 was considered statistically significant.

\section{ACKNOWLEDGMENTS}

None

\section{CONFLICTS OF INTEREST}

None of the authors has any conflicting interests to declare.

\section{GRANT SUPPORT}

This research project was supported by the Funds for International Cooperation and Exchange of the National Natural Science Foundation of China (81020108017), the National Health and Family Planning Commission, China(201302015), the National Major Scientific Equipment program, the Ministry of Science and Technology, China (2012YQ12008003), the State Key Program of National Natural Science Foundation of China (81430007), and the New Technology Research Project, Shanghai Municipal Commission of Health and Family Planning (2013SY058).

\section{REFERENCES}

1. Weinreb RN, Aung T, Medeiros FA. The pathophysiology and treatment of glaucoma: a review. JAMA. 2014; 311:1901-1911.

2. Tham R.C, Li X, Wong TY, Quigley HA, Aung T, Cheng CY. Global prevalence of glaucoma and projections of glaucoma burden through 2040: a systematic review and meta-analysis. Ophthalmology. 2014; 121:2081-2090.

3. Quigley HA, Broman AT. The number of people with glaucoma worldwide in 2010 and 2020. Br J Ophthalmol. 2006; 90:262-267.

4. Narayanaswamy A, Baskaran M, Perera SA, Nongpiur ME, Htoon HM, Tun TA, Wong TT, Goh D, Su DH, Chew PT, Ho CL, Aung T. Argon Laser Peripheral Iridoplasty for Primary Angle-Closure Glaucoma: A Randomized Controlled Trial. Ophthalmology. 2016; 123:514-521.

5. He M, Foster PJ, Johnson GJ, Khaw PT. Angle-closure glaucoma in East Asian and European people. Different diseases? Eye (Lond). 2006; 20:3-12.

6. Nongpiur ME, Ku JY, Aung T. Angle closure glaucoma: a mechanistic review. Curr Opin Ophthalmol. 2011; 22:96-101.

7. Chang D, Sha Q, Zhang X, Liu P, Rong S, Han T, Liu P, Pan $\mathrm{H}$. The evaluation of the oxidative stress parameters in patients with primary angle-closure glaucoma. PLOS One. 2011; 6:e27218.

8. Demirdogen BC, Ceylan OM, Isikoglu S, Mumcuoglu T, Erel O. Evaluation of oxidative stress and paraoxonase phenotypes in pseudoexfoliation syndrome and pseudoexfoliation glaucoma. Clin Lab. 2014; 60:79-86.

9. Izzotti A, Bagnis A, Sacca SC. The role of oxidative stress in glaucoma. Mutat Res. 2006; 612:105-114.

10. Sacca SC, Izzotti A, Rossi P, Traverso C. Glaucomatous outflow pathway and oxidative stress. Exp Eye Res. 2007; 84:389-399.

11. Tezel G. The immune response in glaucoma: a perspective on the roles of oxidative stress. Exp Eye Res. 2011; 93:178-186.

12. Rieck J. The pathogenesis of glaucoma in the interplay with the immune system. Invest Ophthalmol Vis Sci. 2013; 54:2393-2409.

13. Lei Y, Zhang X, Song M, Wu J, Sun X. Aqueous Humor Outflow Physiology in NOS3 Knockout Mice. Invest Ophthalmol Vis Sci. 2015; 56:4891-4898.

14. Lei Y, Stamer WD, Wu J, Sun X. Oxidative stress impact on barrier function of porcine angular aqueous plexus cell monolayers. Invest Ophthalmol Vis Sci. 2013; 54:4827-4835.

15. Davies KJ, Sevanian A, Muakkassah-Kelly SF, Hochstein P. Uric acid-iron ion complexes. A new aspect of the antioxidant functions of uric acid. Biochem J. 1986; 235:747-754.

16. Whiteman M, Ketsawatsakul U, Halliwell B. A reassessment of the peroxynitrite scavenging activity of uric acid. Ann N Y Acad Sci. 2002; 962:242-259. 
17. Bowman GL, Shannon J, Frei B, Kaye JA, Quinn JF. Uric acid as a CNS antioxidant. J Alzheimers Dis. 2010; 19:1331-1336.

18. Irizarry MC, Raman $\mathrm{R}$, Schwarzschild MA, Becerra LM, Thomas RG, Peterson RC, Ascherio A, Aisen PS. Plasma urate and progression of mild cognitive impairment. Neurodegener Dis. 2009; 6:23-28.

19. Vieru E, Koksal A, Mutluay B, Dirican AC, Altunkaynak Y, Baybas S. The relation of serum uric acid levels with L-Dopa treatment and progression in patients with Parkinson's disease. Neurol Sci. 2016; 37:743-747.

20. Lolekha P, Wongwan P, Kulkantrakorn K. Association between serum uric acid and motor subtypes of Parkinson's disease. J Clin Neurosci. 2015; 22:1264-1267.

21. Babizhayev MA, Yegorov YE. Senescent phenotype of trabecular meshwork cells displays biomarkers in primary open-angle glaucoma. Curr Mol Med. 2011; 11:528-552.

22. Sorkhabi R, Ghorbanihaghjo A, Javadzadeh A, Rashtchizadeh N, Moharrery M. Oxidative DNA damage and total antioxidant status in glaucoma patients. Mol Vis. 2011; 17:41-46.

23. Sacca SC, Pascotto A, Camicione P, Capris P, Izzotti A. Oxidative DNA damage in the human trabecular meshwork: clinical correlation in patients with primary open-angle glaucoma. Arch Ophthalmol. 2005; 123:458-463.

24. Kumar DM, Agarwal N. Oxidative stress in glaucoma: a burden of evidence. J Glaucoma. 2007; 16:334-343.

25. Feilchenfeld Z, Yucel YH, Gupta N. Oxidative injury to blood vessels and glia of the pre-laminar optic nerve head in human glaucoma. Exp Eye Res. 2008; 87:409-414.

26. Kuriyama S, Maruyama Y, Nishio S, Takahashi Y, Kidoguchi S, Kobayashi C, Takahashi D, Sugano N, Hosoya T, Yokoo T. Serum uric acid and the incidence of CKD and hypertension. Clin Exp Nephrol. 2015; 19:1127-1134.

27. Al-khateeb E, Althaher A, Al-khateeb M, Al-Musawi H, Azzouqah O, Al-Shweiki S, Shafagoj Y. Relation between uric acid and Alzheimer's disease in elderly Jordanians. J Alzheimers Dis. 2015; 44:859-865.

28. Green K. Free radicals and aging of anterior segment tissues of the eye: a hypothesis. Ophthalmic Res. 1995; 27:143-149.

29. Yuki K, Murat D, Kimura I, Ohtake Y, Tsubota K. Reducedserum vitamin $\mathrm{C}$ and increased uric acid levels in normaltension glaucoma. Graef Arch Clin Exp. 2010; 248:243-248.

30. Erdurmus M, Yagci R, Atis O, Karadag R, Akbas A, Hepsen IF. Antioxidant status and oxidative stress in primary open angle glaucoma and pseudoexfoliative glaucoma. Currt Eye Res. 2011; 36:713-718.

31. Ferreira SM, Lerner SF, Brunzini R, Evelson PA, Llesuy SF. Oxidative stress markers in aqueous humor of glaucoma patients. Am J Ophthalmol. 2004; 137:62-69.

32. Beit-Yannai E, Trembovler V, Solomon AS. Decrease in reducing power of aqueous humor originating from glaucomatous rabbits. Eye (Lond). 2007; 21:658-664.
33. Tanito M, Kaidzu S, Takai Y, Ohira A. Status of Systemic Oxidative Stresses in Patients with Primary Open-Angle Glaucoma and Pseudoexfoliation Syndrome. PLOS One. 2012; 7(e4968011).

34. Astafurov K, Elhawy E, Ren L, Dong CQ, Igboin C, Hyman L, Griffen A, Mittag T, Danias J. Oral microbiome link to neurodegeneration in glaucoma. PLOS One. 2014; 9:e104416.

35. Vohra R, Tsai JC, Kolko M. The role of inflammation in the pathogenesis of glaucoma. Surv Ophthalmol. 2013; 58:311-320.

36. Zeng J, Liu H, Liu X, Ding C. The Relationship Between Helicobacter pylori Infection and Open-Angle Glaucoma: A Meta-Analysis. Invest Ophthalmol Vis Sci. 2015; 56:5238-5245.

37. Kim JM, Kim SH, Park KH, Han SY, Shim HS. Investigation of the Association between Helicobacter pylori Infection and Normal Tension Glaucoma. Invest Ophthalmol Vis Sci. 2011; 52:665-668.

38. Squadrito GL, Cueto R, Splenser AE, Valavanidis A, Zhang H, Uppu RM, Pryor WA. Reaction of uric acid with peroxynitrite and implications for the mechanism of neuroprotection by uric acid. Arch Biochem Biophys. 2000; 376:333-337.

39. Izzotti A, Sacca SC, Longobardi M, Cartiglia C. Sensitivity of ocular anterior chamber tissues to oxidative damage and its relevance to the pathogenesis of glaucoma. Invest Ophthalmol Vis Sci. 2009; 50:5251-5258.

40. Izzotti A, Sacca SC, Cartiglia C, De Flora S. Oxidative deoxyribonucleic acid damage in the eyes of glaucoma patients. Am J Med. 2003; 114:638-646.

41. Hooper DC, Scott GS, Zborek A, Mikheeva T, Kean RB, Koprowski H, Spitsin SV. Uric acid, a peroxynitrite scavenger, inhibits CNS inflammation, blood-CNS barrier permeability changes, and tissue damage in a mouse model of multiple sclerosis. FASEB J. 2000; 14:691-698.

42. Scott GS, Hooper DC. The role of uric acid in protection against peroxynitrite-mediated pathology. Med Hypotheses. 2001; 56:95-100.

43. Yu ZF, Bruce-Keller AJ, Goodman Y, Mattson MP. Uric acid protects neurons against excitotoxic and metabolic insults in cell culture, and against focal ischemic brain injury in vivo. J Neurosci Res. 1998; 53:613-625.

44. Llull L, Amaro S, Chamorro A. Administration of Uric Acid in the Emergency Treatment of Acute Ischemic Stroke. Curr Neurol Neurosci Rep. 2016; 16:4.

45. Wang YX, Xu L, Yang H, Jonas JB. Prevalence of glaucoma in North China: the Beijing Eye Study. Am J Ophthalmol. 2010; 150:917-924.

46. Cheng JW, Zong Y, Zeng YY, Wei RL. The prevalence of primary angle closure glaucoma in adult Asians: a systematic review and meta-analysis. PLOS One. 2014; 9:e103222. 
47. Hong J, Yang Y, Wei A, Deng SX, Kong X, Chen J, Girard MJ, Mari JM, Xu J, Sun X. Schlemm's canal expands after trabeculectomy in patients with primary angle-closure glaucoma. Invest Ophthalmol Vis Sci. 2014; 55:5637-5642.

48. Foster PJ, Buhrmann R, Quigley HA, Johnson GJ. The definition and classification of glaucoma in prevalence surveys. Br J Ophthalmol. 2002; 86:238-242.

49. Atalay E, Nongpiur ME, Yap SC, Wong TT, Goh D, Husain R, Perera SA, Aung T. Pattern of Visual Field Loss in Primary Angle-Closure Glaucoma Across Different Severity Levels. Ophthalmology. 2016; 123:1957-1964.

50. Pillunat KR, Hermann C, Spoerl E, Pillunat LE. Analyzing biomechanical parameters of the cornea with glaucoma severity in open-angle glaucoma. Graef Arch Clin Exp. 2016; 254:1345-1351.

51. Thakkinstian A, Anothaisintawee T, Chailurkit L, Ratanachaiwong W, Yamwong S, Sritara P, Ongphiphadhanakul B. Potential causal associations between vitamin $\mathrm{D}$ and uric acid: Bidirectional mediation analysis. Sci Rep-UK. 2015; 5.

52. Duan Y, Liang W, Zhu L, Zhang T, Wang L, Nie Z, Chen Y, He L, Jin Y, Yao Y. Association between serum uric acid levels and obesity among university students (China). Nutr Hosp. 2015; 31:2407-2411. 